\title{
The influence of cultivation methods on Shewanella oneidensis physiology and proteome expression
}

\author{
Dwayne A. Elias · Sandra L. Tollaksen · David W. Kennedy $\cdot$ Heather M. Mottaz • \\ Carol S. Giometti · Jeffrey S. McLean · Eric A. Hill • Grigoriy E. Pinchuk • \\ Mary S. Lipton · James K. Fredrickson · Yuri A. Gorby
}

Received: 13 June 2007 / Revised: 31 August 2007 / Accepted: 24 October 2007 / Published online: 21 November 2007

(C) The Author(s) 2007

\begin{abstract}
High-throughput analyses that are central to microbial systems biology and ecophysiology research benefit from highly homogeneous and physiologically welldefined cell cultures. While attention has focused on the technical variation associated with high-throughput technologies, biological variation introduced as a function of cell cultivation methods has been largely overlooked. This study evaluated the impact of cultivation methods, controlled batch or continuous culture in bioreactors versus shake flasks, on the reproducibility of global proteome measurements in Shewanella oneidensis MR-1. Variability in dissolved oxygen concentration and consumption rate, metabolite profiles, and proteome was greater in shake flask than controlled batch or chemostat cultures. Proteins indicative of suboxic and anaerobic growth (e.g., fumarate reductase and decaheme $c$-type cytochromes) were more abundant in cells from shake flasks compared to bioreactor
\end{abstract}

Communicated by Ercko Stackebrandt.

\section{A. Elias}

Department of Biochemistry,

University of Missouri-Columbia, Columbia, MO 65211, USA

S. L. Tollaksen · C. S. Giometti

Biosciences Division, Argonne National Laboratory,

Argonne, IL 60439, USA

D. W. Kennedy · H. M. Mottaz · J. S. McLean - E. A. Hill ·

G. E. Pinchuk · M. S. Lipton · J. K. Fredrickson

Biological Sciences Division,

Pacific Northwest National Laboratory,

Richland, WA 99353, USA

Y. A. Gorby ( $\square)$

J. Craig Venter Institute, 11149 North Torrey Pines Road,

Suite 220, La Jolla, CA 92093, USA

e-mail: ygorby@venterinstitute.org; ygorby@jcvi.org cultures, a finding consistent with data demonstrating that "aerobic" flask cultures were $\mathrm{O}_{2}$ deficient due to poor mass transfer kinetics. The work described herein establishes the necessity of controlled cultivation for ensuring highly reproducible and homogenous microbial cultures. By decreasing cell to cell variability, higher quality samples will allow for the interpretive accuracy necessary for drawing conclusions relevant to microbial systems biology research.

Keywords Controlled cultivation · Systems biology · Proteomics $\cdot$ Shewanella

\section{Introduction}

The cultivation of microorganisms has been performed for more than a century beginning with Louis Pasteur (1879), Robert Koch (1881) and R. J. Petri (1882) (Sedgwick 1916; Gest 1987), with ever increasingly sophisticated methods becoming available over time. These methods range from using undefined medium (e.g., boiled meat extracts) in shake flask cultures where growth rate and substrate utilization (Narang et al. 1997) as well as proteomic profiles (Valentine et al. 2005; Wunschel et al. 2005) can vary widely, to defined culturing media combined with state-of-the-science bioreactors (Nethe-Jaenchen and Thauer 1984; Vasconcelos et al. 1994; Elias et al. 2005).

A considerable amount of physiological (Keltjens et al. 1990; Dolla et al. 2000; Elias et al. 2004) and high-throughput functional genomic data (Beliaev et al. 2002; Thompson et al. 2002; Wan et al. 2004) has been generated from microbial samples produced using shake flask batch cultures. Although results may be statistically defensible, the inherent short-comings of this cultivation technique are that essential parameters directly impacting organism physiology 
are poorly described and difficult to control. These parameters include nutrient availability, metabolite production, specific growth rate, and poor mixing leading to cultural/ environmental heterogeneity. Poor mass transfer kinetics can lead to unwanted limitations in dissolved gasses for use by the organism (such as oxygen or hydrogen) and the buildup of gaseous metabolic byproducts, such as $\mathrm{CH}_{4}$ and $\mathrm{CO}_{2}$ that can have secondary effects on cellular metabolism. For example, the accumulation of dissolved $\mathrm{CO}_{2}$ and the production of dissolved organic acids can significantly alter pH in poorly buffered systems (Ji et al. 1995; Narang et al. 1997; Mayville et al. 1999; Miller and Bassler 2001). Fluctuations of $\mathrm{pH}$ in bacterial cultures induce physiological responses in various organisms (Snoep et al. 1990; Blankenhorn et al. 1999; Stancik et al. 2002; Yohannes et al. 2004). Hence, the conditions of cultivation at the beginning of growth can be far different than those when the cells are harvested for analysis. Furthermore, the time of cell harvest during different "phases of growth" will also impact biochemical reaction rates (Wright and Holland 2003) and the complement of cellular proteins (Lipton et al. 2002; Wunschel et al. 2005). In actuality, the functional characteristics and relative abundance of proteins measured are derived from a heterogeneous cell population including cells in various stages of growth that are potentially expressing a range of metabolic states within relatively short time periods. We argue that the term "aerobic shake flask," therefore, is more an operational description than an accurate description of the physiological growth conditions.

In bioreactors, all relevant physiological parameters, including $\mathrm{pH}$, dissolved oxygen $\left(\mathrm{DO}_{2}\right)$, temperature, agitation, and the incoming gas flow rate and composition can be monitored and controlled. Because the nutrient medium can be of variable composition and added to the bioreactor at discreet rates, cells can be cultured with varying degrees of electron donor/carbon source or electron acceptor limitation and over a range of growth rates. The former has important ramifications given the effects of these limitations on cell physiology, while being able to address the issue of different growth rates enables studies that more closely mimic the variable and potentially long generation times typical of many habitats including sedimentary and aquatic environments. A complete mass (fermentation) balance can also be obtained by measuring biogenic gases produced during the cultivation by using in-line mass spectrometry (MS) in addition to the byproducts of carbon and energy source metabolism and the amount of produced biomass. The utilization of such advanced technologies are allowing for unprecedented insights into the metabolic complexities of microbiological systems.

Controlled cultivation technologies, such as chemostats and turbidostats, minimize culture heterogeneity through continual and thorough agitation and by monitoring and controlling all culture parameters. This mixing substantially increases the mass transfer rates of both the influent gas stream and those gases resulting from microbial metabolism, thus creating a more homogenous gas profile throughout the culture (Pin et al. 2002) and reducing the gas effect on $\mathrm{pH}$. Any change in $\mathrm{pH}$ that occurs during cell growth due to non-gaseous metabolic by-products is compensated for by monitoring probes and the addition of acid or base (typically $\mathrm{HCl}$ or $\mathrm{NaOH}$ ) via computer controlled pumps. While there is a greater upfront capital investment for bioreactors, the investment is minor relative to the expense associated with high-throughput functional genomics techniques.

Systems biology seeks to integrate high-throughput and comprehensive analytical techniques such as DNA and RNA microarrays, proteomics and protein interaction analyses, and metabolite measurements with computational biology (i.e., modeling) to describe the structure of the system and responses to individual perturbations. Such knowledge can be used, for example, to predict systems level responses to environmental changes/perturbations. In our opinion, a key element of systems microbiology research is the use of controlled cultivation techniques to generate samples under well-defined conditions where variations in intra- as well as inter-culture variability, are minimized.

The work described herein was designed to quantitatively assess the relative suitability and limitations of various cultivation approaches for systems biology research. Cells cultivated in shake flasks, controlled batch bioreactors and chemostats were subjected to targeted as well as comprehensive proteomic analysis via two-dimensional gel electrophoresis (Beliaev et al. 2002; Abboud et al. 2005) and the MS based accurate mass and time (AMT) tag approach (Conrads et al. 2002; Smith et al. 2002), to determine the extent of biological variation. Recent reports have used controlled cultivation to determine the "metabolic flux" with different carbon, electron donor or nitrogen sources (Daran-Lapujade et al. 2004; Kolkman et al. 2005, 2006; Adams et al. 2006). However, this is the first report documenting the metabolic differences with varied electron-acceptor between cultures. Finally, while others have utilized steady state cultures and cite advantages similar to those mentioned above, this is, to our knowledge, the first report that assesses and quantifies the effect of cultivation methods on microbial physiology and cell proteome.

\section{Materials and methods}

Materials

Acetonitrile and methanol were purchased from Fisher Scientific (Fair Lawn, NJ, USA). Urea, dithiothreitol (DTT), 
and $\mathrm{CaCl}_{2}$ were obtained from Sigma-Aldrich (St Louis, MO, USA) while thiourea, trifluoroacetic acid, 3-[(3-cholamidopropyl) dimethylammonio]-1-propanesulfonate and ammonium bicarbonate were obtained from Aldrich Chemical Company, Inc. (Milwaukee, WI, USA). Sequencing grade, modified trypsin was purchased from Promega (Madison, WI, USA) while ammonium formate was obtained from Fluka (St Louis, MO, USA). Water was purified using a Barnstead Nanopure Infinity water purification system (Dubuque, IA, USA).

Cell cultivation and harvesting

\section{Shake flask cultures}

Shewanella oneidensis MR-1 (ATCC 700550) were cultivated in a defined medium as described elsewhere (Elias et al. 2005) except that $18 \mathrm{mM}$ lactate was used instead of $6 \mathrm{mM}$ and the PIPES concentration was increased to $30 \mathrm{mM}$ since there was no $\mathrm{pH}$ control. Shake flask cultures (0.15 1) were grown aerobically at $30^{\circ} \mathrm{C}$ with constant shaking at $150 \mathrm{rpm}$. Duplicate cultures were inoculated at 2-h intervals over $20 \mathrm{~h}$. After an additional $4 \mathrm{~h}$ of incubation the optical density $\left(\mathrm{OD} ; \mathrm{A}_{600}\right), \mathrm{pH}$ and $\mathrm{DO}_{2}$ were recorded and culture samples for organic acid analysis were taken via syringe, filtered $(0.22 \mu \mathrm{m})$ and stored at $-80^{\circ} \mathrm{C}$ until analyzed. The cultures that had grown for $24 \mathrm{~h}$ were in the late log phase of growth and were then harvested as previously described (Elias et al. 2005). First, $100 \mathrm{ml}$ of culture was centrifuged and the resulting pellet dehydrated for dry weight biomass measurement (MA100 moisture analyzer Sartorius, Inc.; http://www.sartorius.com/). Then, the remaining cells were pelleted by centrifugation $(27,000 \times g$; $\left.8 \mathrm{~min} ; 4^{\circ} \mathrm{C}\right)$. The resulting pellets and approximately $2-$ $3 \mathrm{ml}$ of the supernatant were transferred to cryovials $(1.0 \mathrm{ml})$ and centrifuged $\left(9,000 \times g ; 4 \mathrm{~min} ; 4^{\circ} \mathrm{C}\right)$. After removal of the supernatant, the cryovials containing packed cell pellets were immediately stored at $-80^{\circ} \mathrm{C}$ for later analysis by $2 \mathrm{DE}$ and AMT MS.

\section{Controlled batch cultures}

Cells were cultured in controlled batches using Bioflow model 110 reactors (New Brunswick Scientific, Edison, NJ, USA). For the direct proteome comparison, lactate was used at $18 \mathrm{mM}$ as in the shake flasks but the PIPES concentration was lowered to $3 \mathrm{mM}$ since $\mathrm{pH}$ control was present. Cells were grown with oxygen at $20 \%$ of dissolved saturation $\left(\mathrm{DO}_{2}\right)$ using a combination of air and $\mathrm{N}_{2}$ gas while $\mathrm{pH}$ was constantly maintained at $7.00 \pm 0.03$ by the addition of $2 \mathrm{~N} \mathrm{HCl}$ or $2 \mathrm{~N} \mathrm{NaOH}$. The temperature was a constant $30^{\circ} \mathrm{C}$ and the reactors were stirred at $400 \mathrm{rpm}$ to minimize mass transfer limitations of $\mathrm{O}_{2}$ and $\mathrm{CO}_{2}$. Samples were taken for organic acid analysis as described above until the late log phase of growth at $\mathrm{A}_{600} \sim 0.6$. At this point cells were harvested for the analyses as described above for the shake flasks.

\section{Chemostat cultures}

Steady state cultures were attained using Bioflow model 3000 reactors (New Brunswick Scientific, Edison, NJ, USA) with the same medium and identical parameters and controls as described for the controlled batch cultures. Once the optical density (OD; $\mathrm{A}_{600}$ ) was approximately 0.6 , fresh medium was pumped into the reactor at a dilution rate of $0.1 / \mathrm{h}$ and a constant 31 volume maintained. Culturing continued until a constant OD and acid addition rate (i.e., steady state) was attained. Samples for organic acids were collected periodically and the cells harvested as above.

\section{Organic acid quantitation}

The organic acids lactate, acetate, pyruvate, formate and fumarate were measured using isocratic flow $(0.5 \mathrm{ml} / \mathrm{min})$ with both high performance liquid chromatography (HPLC) and ion chromatography (IC). The HPLC (Agilent 1100 series HPLC with a $300 \times 78 \mathrm{~mm}$ Rezex organic acid column (Phenomenex, Torrance, CA, USA); $60^{\circ} \mathrm{C} ; 35 \mathrm{~min}$ run time) used a dilute acid mobile phase of $0.005 \mathrm{~N}$ $\mathrm{H}_{2} \mathrm{SO}_{4}$. Quantification was accomplished by injecting the sample $(25 \mu \mathrm{l})$ and using either a diode array detector or a fixed wavelength detector at $210 \mathrm{~nm}$ (4 nm bandwidth). Samples were prepared by filtering $1 \mathrm{ml}(0.2 \mu \mathrm{m})$ and acidifying with $10 \mu \mathrm{l}$ of $2.5 \mathrm{~N} \mathrm{H}_{2} \mathrm{SO}_{4}$. Lactate had a retention time of $18.8 \mathrm{~min}$ and detection limit of $\sim 0.1 \mathrm{mM}$ while pyruvate, acetate, and formate retention times were 13.4, 22.1, and $20.2 \mathrm{~min}$, respectively with a detection limit each of $\sim 0.2 \mathrm{mM}$. For IC (Dionex DX-500) analysis was performed for the above organic acids as previously described (Senko et al. 2002; Elias et al. 2004).

\section{D-gel proteomic analysis}

Frozen cell pellets were mixed with 2 vols. of a solution containing $9 \mathrm{M}$ urea, 2\% 2-mercaptoethanol, 2\% ampholytes (pH 8-10, BioRad), and 2\% Nonidet P40 (a nonionic detergent). The cell lysate was then ultracentrifuged $(435,000 \times g ; 10 \mathrm{~min})$ using a Beckman TL100 tabletop ultracentrifuge, and the soluble denatured proteins were recovered in supernatants from centrifugation. Protein concentrations were determined using a modification of the Bradford protein assay (Ramagli and Rodriguez 1985). Aliquots of sample containing $40 \mu \mathrm{g}$ of protein were separated in the first dimension by isoelectric focusing using polyacrylamide gels containing $50 \%$ pH 5-7 with 50\% pH 3-10 
carrier ampholytes (Anderson and Anderson 1978a). After $14,000 \mathrm{~V}$-h, the first-dimension gels were equilibrated with sodium dodecyl sulfate (SDS) and the proteins were separated by SDS-polyacrylamide gel electrophoresis as described (O'Farrell 1975) using a linear gradient of 1017\% acrylamide (Anderson and Anderson 1978b). Proteins were then detected by staining with silver nitrate (Giometti et al. 1991). The 2DE images were digitized using an Eikonix1412 scanner interfaced with a VAX 4000-90 workstation. The images were then transferred to a PC, converted to TIFF format, and then processed for spot detection and pattern matching using the Progenesis software (Nonlinear USA). One 2DE image was used as a reference pattern for the experiment. All patterns in the experiment were matched to the reference pattern so that the protein spots were given identification numbers. Statistical analysis of the relative abundance of each matched protein spot across the data set was done using a two-tailed Student's $t$-test as previously described (Giometti and Taylor 1991). Proteins to be identified were cut from two to three replicate gels stained with Coomassie Blue R250 (approximately $200 \mu \mathrm{g}$ of protein was loaded on each gel), and the proteins were digested in-gel with trypsin (Promega sequence-grade trypsin, $12.5 \mathrm{ng} / \mu \mathrm{g}$ ). The resulting peptides were eluted from the gel pieces by extracting three times, first with equal parts of $25 \mathrm{mM}$ ammonium bicarbonate and acetonitrile, then twice with equal parts of $5 \%(\mathrm{v} / \mathrm{v})$ formic acid and acetonitrile. The eluted tryptic peptides were desalted and concentrated with a commercial ZipTip $\mathrm{C}_{18}$ pipette tip (Millipore). Peptide samples were then loaded onto a $365 \times 100 \mu \mathrm{m}$ fused silica capillary (FSC) column packed with $10 \mu \mathrm{m}$ POROS $10 \mathrm{R} 2$ packing material (PE Biosystem) at a length of $10-15 \mathrm{~cm}$. Peptides were separated with a 30-min linear gradient of $0-60 \%$ solvent containing $80 \%$ acetonitrile and $0.5 \%$ acetic acid, and then entered into an LCQ ion trap mass spectrometer (Finnigan MAT). Tandem mass spectra were automatically collected under computer control during the 30-min LC-MS runs. MS/MS spectra were then directly subjected to SEQUEST database searches (Eng et al. 1994; Sadygov et al. 2002) by correlating experimental MS/MS spectra to predicted protein sequences in the $S$. oneidensis MR-1 open reading frame database.

Mass spectrometry proteomic analysis

\section{Cell lysis and tryptic digestion}

Whole cell lysis was achieved by bead beating while tryptic digestion was achieved with sequencing grade-modified trypsin as previously described (Elias et al. 2005) and digested for $5 \mathrm{~h}$ at $37^{\circ} \mathrm{C}$ using a 1:50 (w/w) trypsin-to-protein ratio. Samples were quick frozen in liquid $\mathrm{N}_{2}$ and stored at $-80^{\circ} \mathrm{C}$ until analyzed. Protein concentration was determined by the BCA assay kit (Pierce, Rockford, IL, USA).

\section{Mass spectrometric analysis}

The capillary LC system used was described previously (Elias et al. 2005) using 5,000 psi reversed-phase packed capillaries at $\sim 1.8 \mu \mathrm{l} / \mathrm{min}(150 \mu \mathrm{m}$ i.d. $\times 360 \mu \mathrm{m}$ o.d.; Polymicro Technologies) (Shen et al. 2001, 2002) with two mobile phase solvents consisting of $0.2 \%$ acetic acid and $0.05 \%$ TFA in water (A) and $0.1 \%$ TFA in $90 \%$ acetonitrile $/ 10 \%$ water (B). The LTQ-FT data was processed using the PRISM data analysis system as described previously for LC-FTICR-MS data (Elias et al. 2005). Since the separation systems for both the LTQ-FT and the LCQ analyses were identical, peptide confirmation was based on both the calculated (from the mass tag database) and measured mass (from the FTICR analysis) of the peptide matching to within $6 \mathrm{ppm}$ and the elution times matching to within 5\%.

All samples were analyzed as previously described (Lipton et al. 2002; Elias et al. 2005) using an LTQ-FT (Thermo-Finnigan, MI, USA). The mass spectrometer measurements were analyzed with SEQUEST (Eng et al. 1994) using our current database as previously described (Elias et al. 2005). Mass spectra were acquired with approximately $10^{5}$ resolution.

The peptide identifications were determined using the spatially localized confidence scoring (SLiC) algorithm (Norbeck et al. 2005) that incorporates a number of constraints and estimates the confidence of each peptide identification by yielding a score of $0-1$. Recent work in our laboratory shows that a SLiC score of at least 0.7 is sufficiently rigorous (unpublished) and is used herein. Further, at least one high-confidence "unique" peptide (i.e., mapping to only one possible parent protein) and a total of two peptides was required for protein identification in each analysis.

\section{Results}

Effect of cultivation method on growth and metabolism

Duplicate cultures were grown in; (1) shake flasks (uncontrolled batch) (2) controlled batch and (3) continuous flow reactors operating as steady state chemostats all with $\mathrm{O}_{2}$ as the sole terminal electron acceptor. Shake flask cultures exhibited an extended lag phase (approximately $13 \mathrm{~h}$ ), which compared with the shorter lag phase $((5 \mathrm{~h})$ in controlled batch cultures (Fig. 1a,b). The extended lag and slow growth rates $((0.15 / \mathrm{h})$ in shake flasks correlated with elevated $\mathrm{DO}_{2}$ concentration that gradually decreased from 
Fig. 1 A comparison of duplicate uncontrolled versus duplicate controlled (right) batch culturing of $S$. oneidensis MR-1. a The inconsistency in decreasing $\mathrm{DO}_{2}$ (dark filled diamond, open diamond) and growth (dark filled triangle, open triangle) in the uncontrolled cultures is apparent when compared to those for controlled batch cultures (b) using the same symbols. This variation is reflected in the (c, d) organic acid profiles. The lactate (dark filled diamond, open diamond), pyruvate (dark filled square, open square) and acetate (dark filled triangle, open triangle) all exhibited more similar profiles between culture replicates in the controlled batches
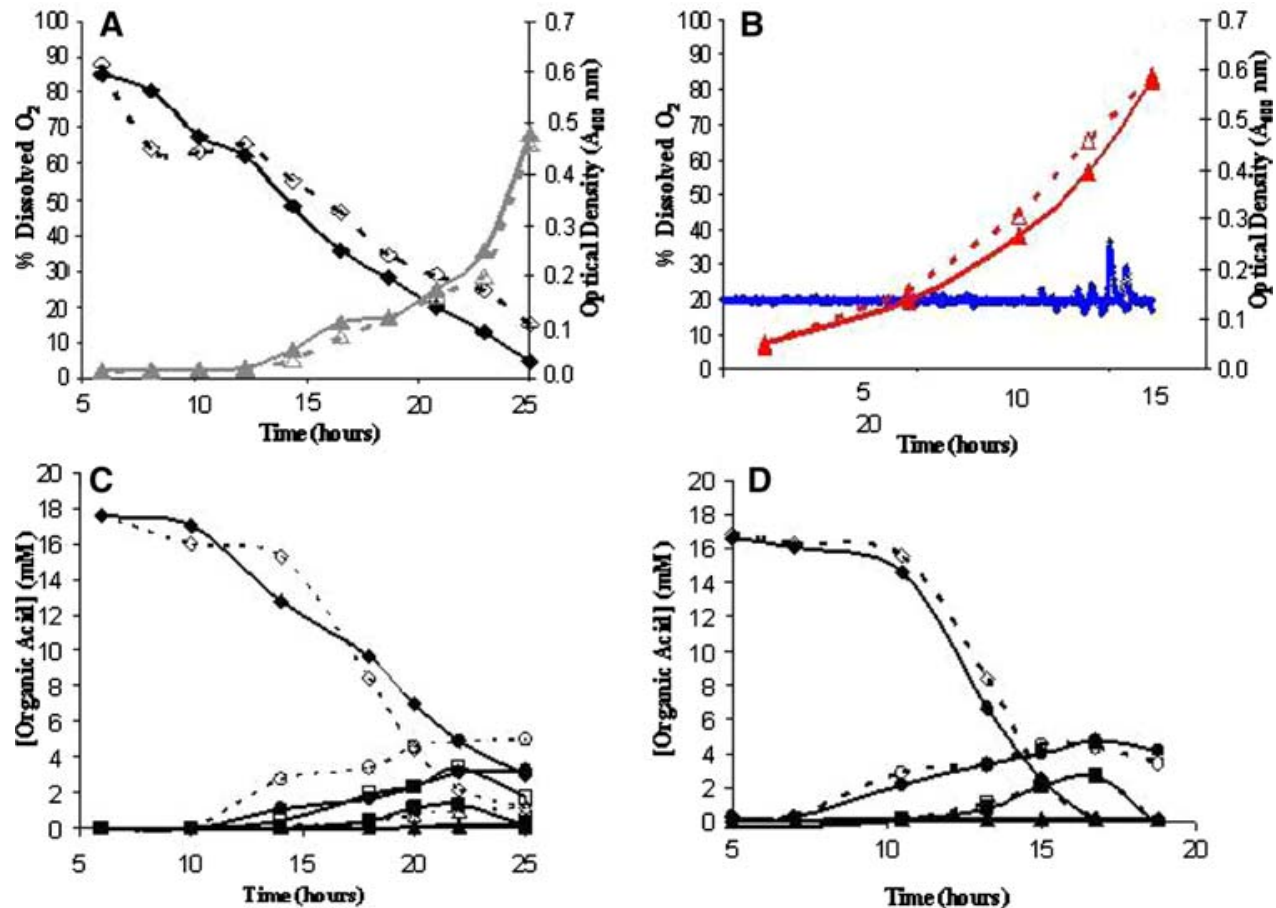

nearly $100 \%$ of air saturation to approximately $20 \%$ of air saturation (Fig. 1a). Below 20\% $\mathrm{DO}_{2}$ growth rates increased to approximately $0.275 / \mathrm{h}$, which is close to the growth rate observed for controlled batch culture operated at DOT $20 \%$ of air saturation (Fig. 1a, b). By definition, chemostat cultures operating at steady state were maintained at a constant growth rate $(0.1 / \mathrm{h})$ and $20 \% \mathrm{DO}_{2}$ throughout the course of the experiment (data not shown).

Despite the similarity in growth rates between the duplicate flask cultures (Fig. 1a), the decrease in $\mathrm{DO}_{2}$, i.e., the $\mathrm{O}_{2}$ consumption rates, were dissimilar. These differences correlated with variations in the organic acid concentration profiles in shake flask cultures (Fig. 1c). Although the order of appearance and disappearance of the organic acids was the same between the flask culture replicates, the rate of lactate consumption varied significantly as did the transient concentrations of pyruvate and acetate. The differences in these profiles presumably reflect the metabolic variability between cultures at any given point along the growth curve, and may result from increased variability in the mass transfer kinetics in poorly mixed cultures compared to controlled batch.

In comparison, maintaining a constant $\mathrm{DO}_{2}$ concentration in the controlled batch cultures resulted in a shorter lag phase with nearly identical growth rates (Fig. 1b). Cells in well-mixed controlled batch cultures were continuously exposed to a constant $20 \% \mathrm{DO}_{2}$ concentration, as opposed to variable $\mathrm{DO}_{2}$ concentrations typical in "aerobic" flask cultures that in fact become $\mathrm{O}_{2}$-limited as cell densities increase due to poor mass transfer rates. This resulted in much greater consistency in organic acid concentration profiles between the replicates (Fig. 1d).

\section{Relating cultivation data to metabolic activity}

Not only does controlled cultivation result in more consistent metabolic activities within and between cultures as shown above, but the very nature of highly instrumented and controlled cultivation provides important characterization data that help to relate cellular metabolism with the environmental conditions experienced by the culture. In a controlled batch experiment designed to illustrate these relationships, cell growth (Fig. 2a) was correlated with the $\mathrm{DO}_{2}$ consumption and the amount of acid needed to maintain a constant pH (Fig. 2b), as well as the organic acid profiles (Fig. 2c). Figure 2a illustrates the increase in optical density measured during the course of the experiment. The solid circles indicate optical density of samples taken directly from the culture, while solid squares represent optical density measurements on the same samples after the addition of $10 \mu \mathrm{M}$ Na-EDTA. This treatment destabilized cell aggregates (flocs) by chelating calcium, which can cross-link polysaccharides in extracellular polymeric substances. The ratio of flocculated:deflocculated cultures is termed the flocculation index (FCI) and is represented by the open triangles. Hence, flocculation indices above 1 indicate the presence of cell aggregates while values at or slightly below 1 indicate the absence of flocculated cells. The cultures lacked flocculated cells during the first $15 \mathrm{~h}$ of growth (Fig. 1a). Acid addition (Fig. 2b, red line) during 

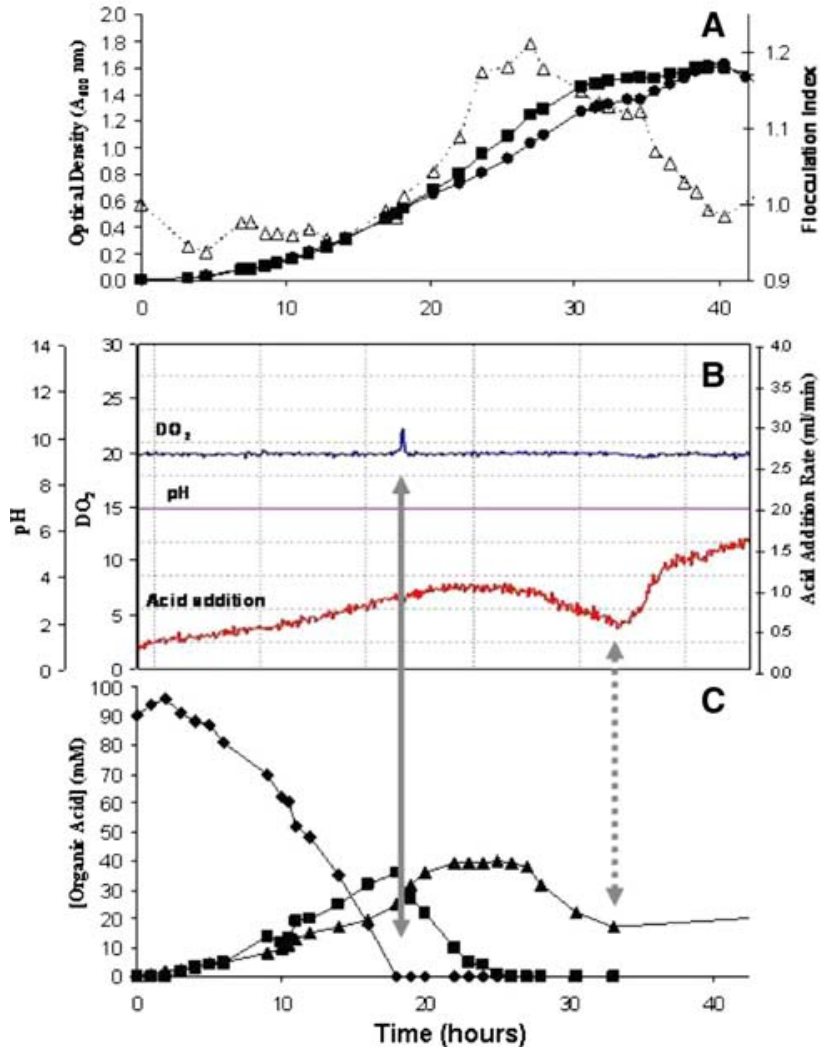

Fig. 2 Alignment of the (a) growth, (b) controlled parameters and (c) organic acid profile for $S$. oneidensis MR-1 cells grown in controlled batch to indicate consequent shifts in metabolism. a The growth of flocculated (dark filled square; after EDTA addition), and non-flocculated cells (dark filled circle) were initially compared to profile the degree of flocculation (dark filled triangle) during growth. When coordinated with changes in (b) $\mathrm{pH}, \mathrm{DO}_{2}$ and acid addition and (c) the appearance and exhaustion of the organic acids lactate (dark filled diamond), pyruvate (dark filled square), acetate (dark filled triangle), changes in metabolism can be identified. These include the $\mathrm{DO}_{2}$ spike (solid arrow) signaling lactate exhaustion with the concomitant increase in cell floculation as well as the decreased acid addition rate (broken arrow) showing major carbon source exhaustion and the concomitant disappearance of flocculation

this period directly tracked increases in optical density. Lactate served as the primary electron donor and carbon source, and decreased while pyruvate and acetate accumulated in the medium (Fig. 2c).

The point at which lactate was completely consumed (18 h, solid gray arrow) corresponded with a transient spike in $\mathrm{DO}_{2}$ (Fig. 2b, blue line). This peak resulted from a rapid decrease in $\mathrm{O}_{2}$ consumption rate and a delayed response of the control loop used by the reactor to maintain constant $\mathrm{DO}_{2}$ values. The coordinated decrease in respiration rate and the complete consumption of lactate as the primary electron donor also correlated with the onset of flocculation, as indicated by an increased FCI. The organic acid analyses clearly indicated that pyruvate assumed the role of primary electron donor with no apparent change in acetate concentration. Cell flocculation peaked at $\sim 25 \mathrm{~h}$, just as the last of detectable pyruvate was consumed. The subsequent decrease in the FCI was bimodal. The initial decrease in the FCI corresponded to a slow decline in alkalinity production as measured by decreasing acid addition to maintain $\mathrm{pH}$. When the concentration of acetate fell to $\sim 15 \mathrm{mM}$ and the FCI entered its second phase and decreased sharply, acid addition increased dramatically. This was likely a result of both the decrease in microbial metabolism and the aeration of the bioreactor. During active growth, lactate will be mineralized to both $\mathrm{CO}_{2}$ and bicarbonate with the $\mathrm{CO}_{2}$ being stripped from the culture via aeration and bicarbonate modestly increasing the alkalinity of the culture. As the metabolic rate of the culture decreases due to nutrient limitation, $\mathrm{CO}_{2}$ and bicarbonate generation will also decrease, but the $\mathrm{CO}_{2}$ will continue to be removed from the system while increasing amounts of bicarbonate will form carbonic acid and $\mathrm{CO}_{2}$. This will result in a net loss of protons from the system, thus increasing the $\mathrm{pH}$ and cause the increased addition of acid to the culture.

\section{Correlating protein expression with culture conditions}

Significant differences were observed between the proteomes of cells from uncontrolled "aerobic" flasks and controlled aerobic batch reactors when analyzed by $2 \mathrm{DE}$ and MS. In general, proteins predicted to be expressed in cells growing under low oxygen tensions or anaerobically [e.g., fumarate reductase (Myers and Myers 1992; Maier et al. 2003], OmpW, glutamine synthase, decaheme cytochrome $c$ MtrA, MtrC, and OmcA (Beliaev et al. 2002, 2005; Fang et al. 2006) were exclusively expressed or detected with increased abundance in samples from shake flasks when compared to those from controlled batch and chemostat cultures. Fumarate reductase (spot 432, SO0970), an enzyme required for anaerobic growth with fumarate (Maier et al. 2003), was detected in high abundance in 2DE patterns of lysate proteins from cells grown in the flask cultures, but was not detected in samples from aerobic controlled batch reactors (Fig. 3). Similarly, the outer membrane protein OmpW (spot 1945, SO1673), and a hypothetical protein (spot 1275, SO3549) were present in 2DE patterns of proteins from flask culture lysates, but not detected in aerobic controlled batch reactors. A significant increase in the number of peptides corresponding to OmpW and fumarate reductase $(8.1 \times$ and $6.3 \times$ higher, respectively, in the flask samples compared to controlled batch samples) was observed in the MS-based AMT tag analyses of these lysates (Table 1), confirming the 2DE results. It is presently unclear why OmpW and the hypothetical protein abundances increased in low $\mathrm{DO}_{2}$, but our laboratory has observed this trend in the former protein several times (unpublished). In addition, peptides representing the multiheme cytochromes 
Fig. 3 Two-dimensional gel electrophoresis patterns of whole cell lysate proteins from S. oneidensis MR-1 grown in shake flasks or continuous batch cultures. The proteins indicated by spot number correspond to those identified as varying with statistical significance between the two different culture methods (see Table 1). The gel images are oriented with the basic proteins to the right, acidic proteins to the left, high molecular weight toward the top and low molecular weight toward the bottom. Flask: shake flask cultures (two replicate experiments); CB, controlled batch cultures (two replicate experiments)
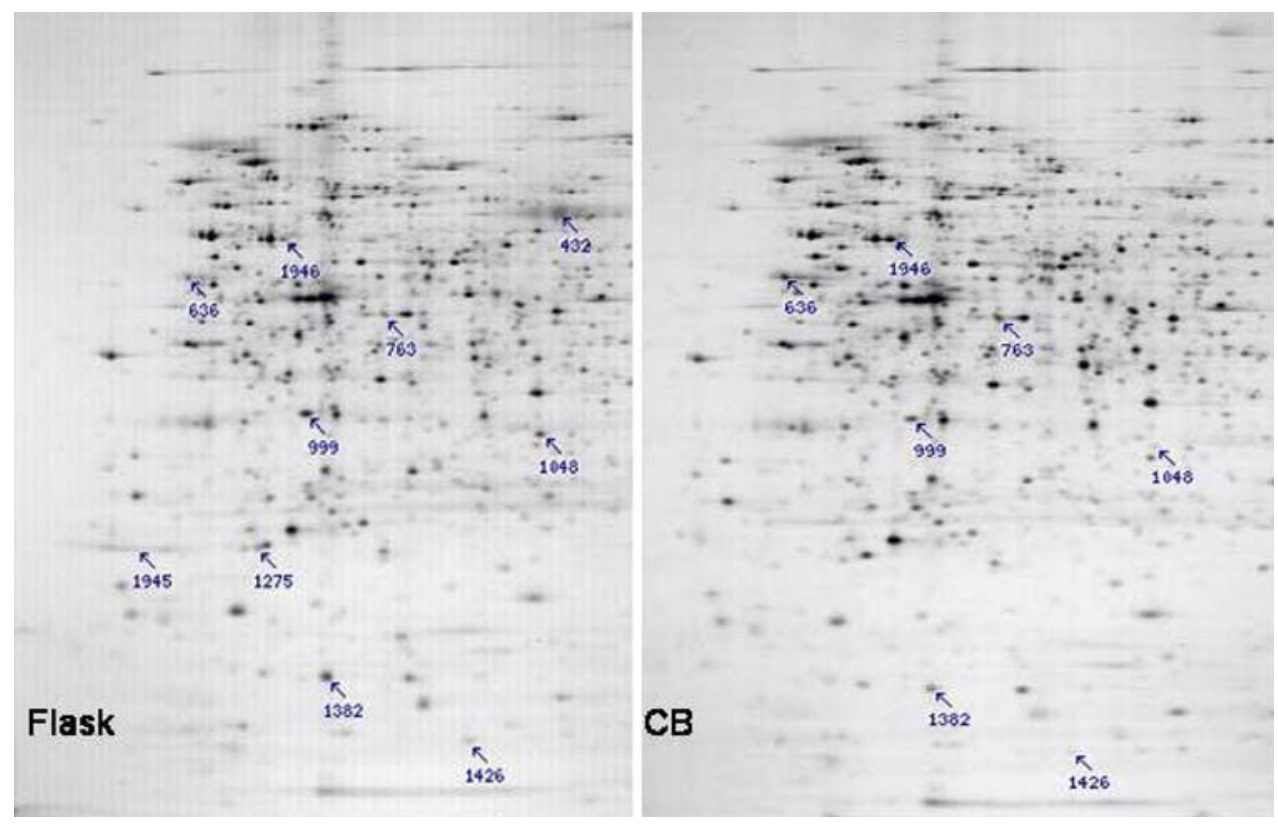

Table 1 Differential protein detection between flask and controlled batch cultures as determined by 2DE and MS

\begin{tabular}{|c|c|c|c|c|c|c|c|c|c|}
\hline \multirow[t]{2}{*}{ ORF } & \multirow[t]{2}{*}{ Protein name } & \multirow{2}{*}{$\begin{array}{l}\text { Spot } \\
\text { number }\end{array}$} & \multicolumn{4}{|l|}{$2 \mathrm{DE}$} & \multicolumn{3}{|l|}{ MS } \\
\hline & & & Avg flask & Avg CB & $P$-value & Flask/CB ratio & Avg flask & Avg CB & Flask/CB ratio \\
\hline SO0970 & Fumarate reductase & 432 & 4900 & ND & - & - & $106 \pm 34$ & $17 \pm 2^{\mathrm{b}}$ & 6.3 \\
\hline SO4349 & Ketol-acid reductoisomerase & 636 & 1148 & 3150 & 0.003 & 0.4 & $22 \pm 6$ & $139 \pm 26$ & 0.2 \\
\hline SO1931 & 2-Oxoglutarate dehydrogenase & 763 & $495^{\mathrm{a}}$ & 951 & $0.024^{\mathrm{a}}$ & 0.5 & $36 \pm 1$ & $46 \pm 4$ & 0.8 \\
\hline SO3237 & Flagellin & 999 & 2711 & 1223 & 9.9E-05 & 2.2 & $108 \pm 36$ & $73 \pm 14$ & 1.5 \\
\hline- & ND & 1048 & 1196 & 196 & $8.8 \mathrm{E}-04$ & 6.1 & - & - & - \\
\hline SO3549 & Hypothetical protein & 1275 & 648 & ND & - & - & - & - & - \\
\hline SO3681 & Universal stress protein family & 1382 & 2600 & 701 & $3.5 \mathrm{E}-06$ & 3.7 & $51 \pm 12$ & $13 \pm 2$ & 3.9 \\
\hline- & ND & 1426 & 313 & ND & - & - & - & - & - \\
\hline SO1673 & OmpW & 1945 & 2927 & ND & - & - & $62 \pm 24$ & $8 \pm 1$ & 8.1 \\
\hline SO4410 & Glutamine synthetase, type I & 1946 & 1001 & 1747 & $1.8 \mathrm{E}-04$ & 0.6 & $58 \pm 12$ & $63 \pm 3$ & 0.9 \\
\hline SO1776 & MtrB & & & & & & $7 \pm 1$ & $4 \pm 1$ & 2.1 \\
\hline SO1777 & Decaheme cytochrome $c$ MtrA & & & & & & $8 \pm 1$ & $2 \pm 0$ & 5.5 \\
\hline SO1778 & Decaheme cytochrome $c$ MtrC & & & & & & $36 \pm 6$ & $18 \pm 1$ & 2.0 \\
\hline SO1779 & Decaheme cytochrome $c$ OmcA & & & & & & $21 \pm 3$ & $11 \pm 1$ & 1.9 \\
\hline
\end{tabular}

ORF open reading frame (www.ncbi.nih.gov/), Protein name annotation for open reading frame (www.ncbi.nih.gov/), Spot number spot number assigned by Progenesis software using images shown in Figs. 3 and 5, Avg flask average integrated spot density in 2DE images (3-4 images per sample) from shake flask samples, $A v g C B$ average integrated spot density in 2DE images (3-4 images per sample) from continuous batch culture samples, $P$-value $P$-value from two-tailed Student's $t$-test analysis of average integrated density values, Flask/CB ratio ratio of average integrated density values for flask and continuous batch culture samples

${ }^{a}$ Note that the difference in spot 763 is a function of the variability in the protein between the two flask cultures. In Flask 1, the average integrated density was 1020 with a coefficient of variation (CV) of $12.5 \%$ whereas in Flask 2, the average integrated density was 316 with a CV of $31.6 \%$. The 495 represents the averaging of integrated densities from all of the patterns from these two flasks together with a resulting CV of $60 \%$

b The average MS abundance data \pm the standard deviation resulting from triplicate analysis of each biological sample

MtrA, MtrC, and OmcA and outer membrane $\beta$-barrel protein MtrB, were observed in two- to fivefold higher abundance in the AMT tag analysis of samples from shake flasks versus those from controlled batch cultures. These observa- tions strongly suggest the use of shake flask cultures to assess changes in protein expression patterns related to aerobic versus anaerobic metabolism could yield inaccurate results. 


\section{Chemostats provide enhanced experimental consistency}

Chemostat cultures provide the highest level of control of any known cultivation technology (Monod 1950; Novick and Szilard 1950; Wimpenny 1985). To evaluate the reproducibility of chemostat cultures, duplicate bioreactors were operated in steady state with S. oneidensis MR-1 under $50 \% \mathrm{DO}_{2}$ as above and the growth and carbon metabolism parameters were measured. Each of the aerobic cultures attained an initial maximum optical density of $\sim 0.70$ by $20 \mathrm{~h}$ and eventually achieved a steady state cell density at $0.53\left(\mathrm{~A}_{600 \mathrm{~nm}}\right)$ (Fig. 4). The two reactors were highly similar in terms of growth and acid addition rates, as well as organic acid concentrations at the time of sampling of 100 h. Lactate, acetate, fumarate and succinate were all below detectable limits suggesting that the cultures were in fact carbon-limited during steady state growth. Pyruvate was present in both chemostatic cultures at $0.14 \pm$ $0.02 \mathrm{mM}$. Dry weight biomass measurements confirmed the highly similar cell densities of $338.0 \pm 4.3 \mathrm{mg} / \mathrm{l}$ with only a $1.3 \%$ variance between the reactors. In comparison, the duplicate controlled batch and shake flask cultures used in this study yielded similar biomass amounts but varied by 2.3 and $17.6 \%$, respectively. These results support our contention of greater biological variability among replicate flask cultures relative to bioreactor cultures.

\section{Culture and proteome variability}

When the intercultural variability of the proteomes of cells from duplicate shake flasks, controlled batch bioreactors, and chemostat cultures was assessed using 2DE and MS, notable differences were identified. For 2DE analysis, digital images of the gels were processed for spot detection and pattern matching with one $2 \mathrm{DE}$ image as a reference pattern and all others matched to it to give the protein spots identification numbers. Statistical analysis of the relative abundance of each matched protein spot across the datasets employed a two-tailed Student's $t$-test as previously described (Giometti and Taylor 1991). Differences in 2DE spot patterns were observed between the replicate flask cultures (Fig. 5a, d), including one unidentified protein (spot $771 ; P<0.004)$. In contrast, there was relatively little variability $(P<0.05)$ in $2 \mathrm{DE}$ proteome profiles from the replicate gels from either the controlled batch or chemostat cultures.

Data from MS-proteome analysis was consistent with the $2 \mathrm{DE}$ results. Triplicate samples from duplicate cultures from shake flask, controlled batch, and chemostats were analyzed and Venn diagrams (Fig. 6) were constructed to illustrate the similarities and differences between biological and analytical replicates based on the cultivation method. A comparison of protein detection consistency between the biological duplicates showed that the flask grown cells (Fig. 5a) exhibit a higher degree of variability. Flask A averaged 972 proteins detected from triplicate analyses while flask B averaged 1,029 proteins with only 770 proteins $(77 \%)$ in common. The consistency of detected proteins between biological replicates was higher in the controlled batch (84\%, Fig. 5c) and chemostat cultures (88\%, Fig. 5e). This was not due to a lack of consistency in the analytical replicates since protein detection consistency was between 88 and $89 \%$ for all three cultivation methods (Fig. 5d-f). Secondly, there was an overall decrease in the
Fig. 4 Shewanella oneidensis MR-1 cultured in duplicate bioreactors at $20 \% \mathrm{DO}_{2}$. The same inoculum culture was used for both bioreactors. Cells were harvested from each reactor when the optical density and organic acid concentrations were similar. The average dry weight biomass was $338 \pm 4.3 \mathrm{mg} / \mathrm{l}$

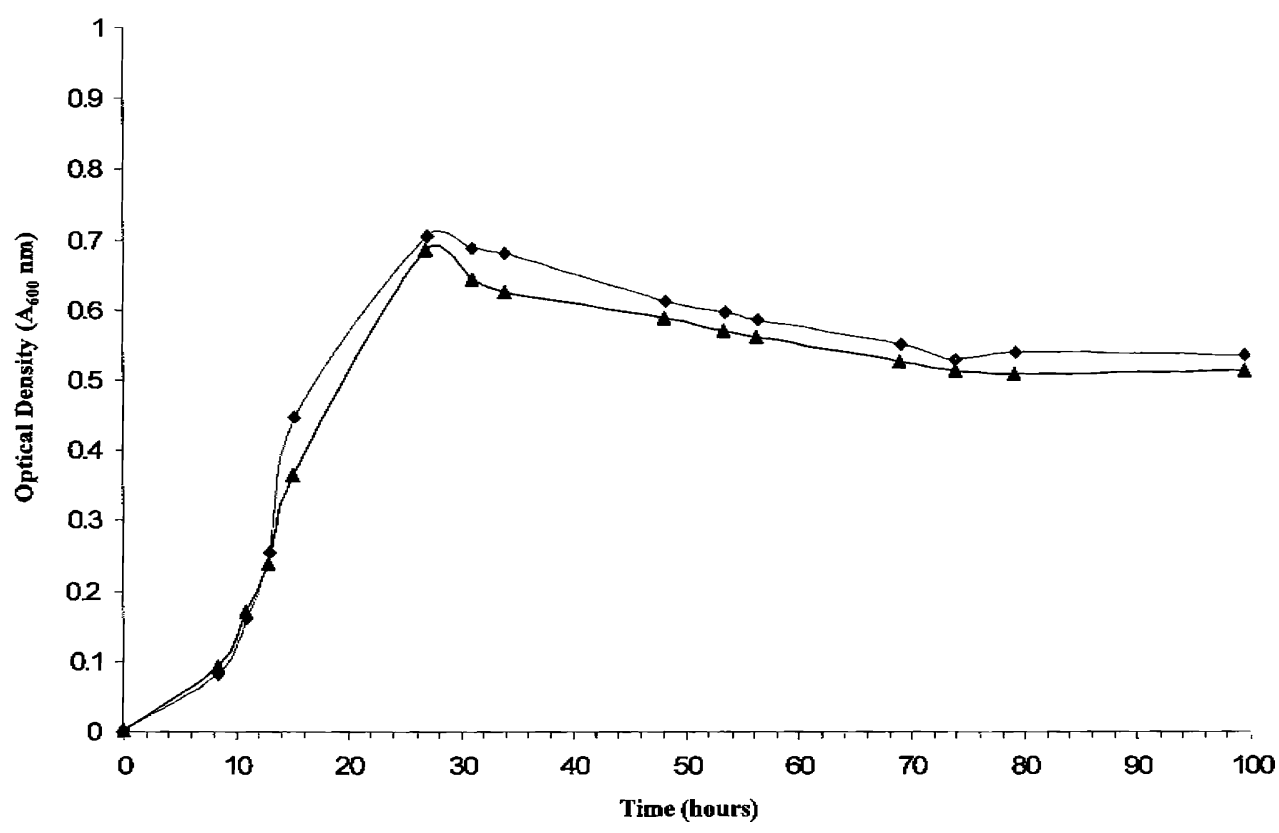


Fig. 5 2DE protein patterns from biological replicates of the three growth methods. The within method replicates show few significant differences. The chemostat and controlled batch replicates showed no quantitative differences with a statistical significance of $P<0.05$ whereas the flask culture replicates showed one quantitative difference (Spot 771) with a difference of $P<0.004$. SS steady state culture, $C B$ controlled batch culture. The gel images are oriented with the basic proteins to the right, acidic proteins to the left, high molecular weight toward the top and low molecular weight toward the bottom
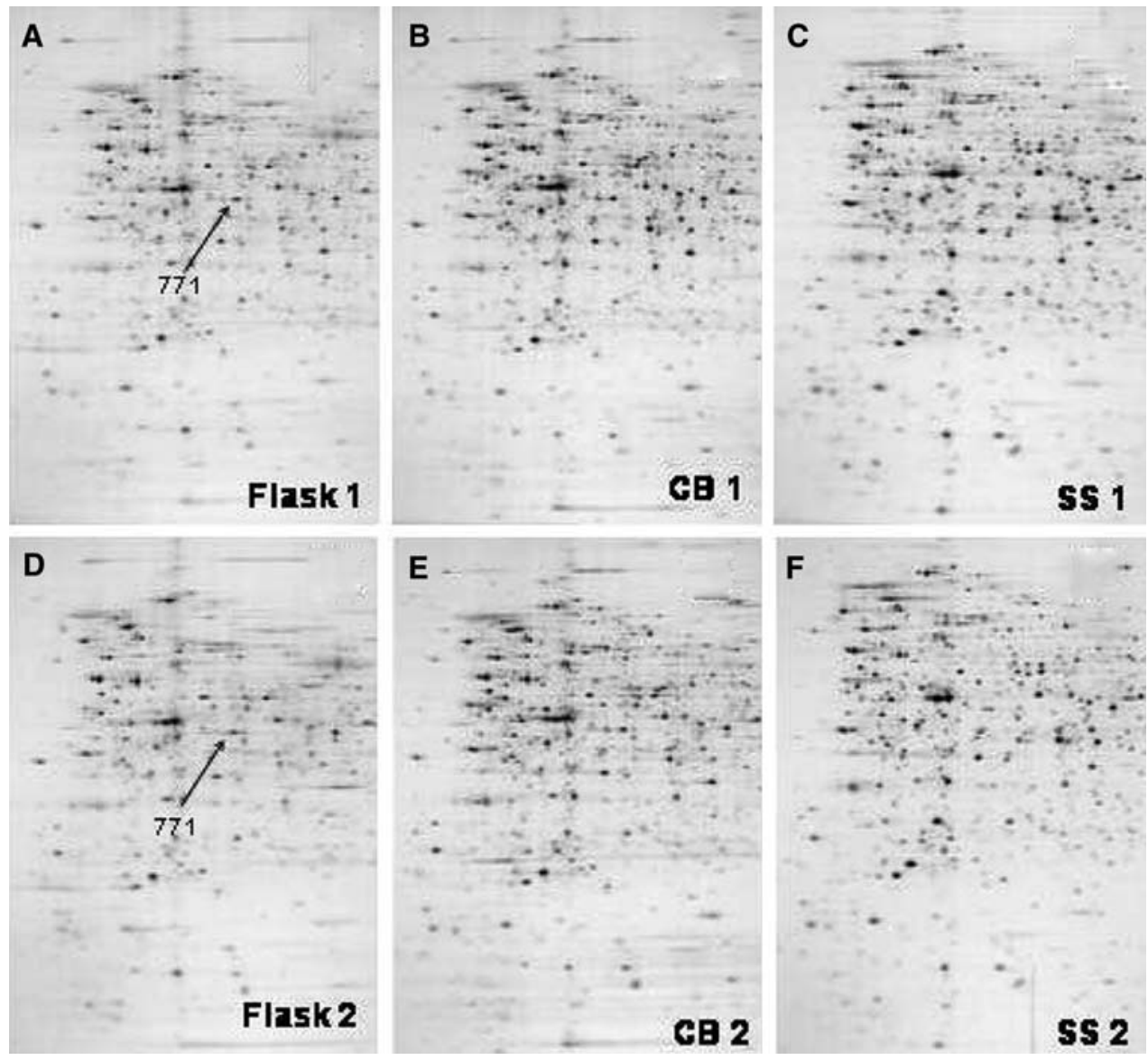

number of proteins detected from the flask grown (770) to the controlled batch (713) and to the chemostat (571) cultures. This can be explained by the presumed homogeneity of the culture increasing as the degree of control over $\mathrm{pH}$ and $\mathrm{DO}_{2}$ increased thus allowing the entire culture to experience similar environmental conditions.

\section{Discussion}

The advantages of controlled cultivation have been demonstrated for many different research applications in microbiology, including the determination of carbon, nitrogen or electron flow (Vasconcelos et al. 1994; Daran-Lapujade et al. 2004; Kolkman et al. 2005, 2006; Adams et al. 2006), kinetic constants for specific reactions (Nethe-Jaenchen and Thauer 1984), alternate roles for traditionally well-defined enzymes (Snoep et al. 1990), and whole cell protein fingerprinting (Wunschel et al. 2005). While there have been statements touting the advantages of controlled cultivation, an in depth investigation of the differences in metabolism between cultivation methods has not been performed. The closest exception to this reported that microarray results between three chemostatic cultures from three different laboratories were significantly more reproducible compared to a previous study that performed a similar comparison using shake flask cultures (Piper et al. 2002). However, the authors reported that there were differences in harvesting and other procedures which may well have skewed the findings. To this end, the experiments reported here were specifically directed toward determining whether or not controlled cultivation results in a more homogenous culture with regard to both targeted and comprehensive proteome profiles from three cultivation methods in our laboratory. Our results demonstrate that variations in $\mathrm{DO}_{2}$ concentration and consumption rates between replicate flask cultures in the late log phase of growth can impact metabolism. This was evidenced by the increased lag time in the uncontrolled flask cultures relative to the controlled cultivations. The data suggests that this may be an effect of oxygen toxicity when $\mathrm{DO}_{2}$ levels are above $20 \%$. This has been recently tested in $S$. oneidensis MR-1 using sealed culturing vessels and levels above $21 \%$ headspace $\mathrm{O}_{2}$ were found to be inhibitory (McLean et al. 2007). Two possible explanations exist for this observation. It is possible that the extended lag and initial slow growth rate was the result of oxygen toxicity at $\mathrm{DO}_{2}$ values above $20 \%$. However, the uncontrolled batch cultures contained $30 \mathrm{mM}$ PIPES buffer 

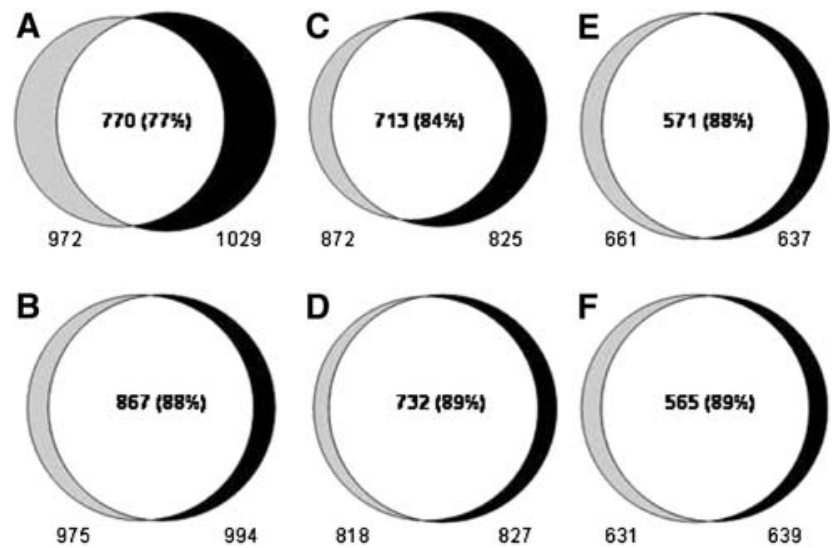

Fig. 6 MS proteomic analysis of the same duplicate (left) shake flask, (middle) controlled batch and (right) chemostat grown cells that were analyzed by $2 \mathrm{DE}$. The consistency in the proteins detected between biological duplicates from shake flask (a), controlled batch (c) and chemostat (e) cultures shows the increased similarity in proteomes with increased control over culturing parameters. This was not an effect of the instrumentation since analytical replicates from each of the shake flask (b), controlled batch (d) and chemostat (f) cultures consistently had (88\% of the detected proteins in common. The values below each Venn diagram circle are the number of detected proteins for each biological $(\mathbf{a}, \mathbf{c}, \mathbf{e})$ or analytical $(\mathbf{b}, \mathbf{d}, \mathbf{f})$ replicate. The value in the overlap region is the number of proteins in common between the replicates with the corresponding percentage for ease of comparison. The percentage shown is the number of proteins in common divided by the average of the two replicates presented

to aid in the maintenance of $\mathrm{pH}$ during growth while the controlled batch and chemostatic cultures contained only $3 \mathrm{mM}$ PIPES. Hence, there would have been a large difference in the ionic strength of the two media and so while $\mathrm{DO}_{2}$ toxicity may be the reason for the extended lag phase, other reasons cannot be ruled out. Separately, differences in acetate concentrations of the replicate shake flask cultures compared to controlled batch and chemostat cultures indicated that such differences may well have promoted additional biological variability.

The difference in acetate concentrations between replicate shake flask cultures compared to the consistent acetate concentrations in controlled batch and chemostat cultures has particular implications for $S$. oneidensis strain MR-1. MR-1 can use lactate and pyruvate as an electron donor during anaerobic growth, but not acetate (Lovley et al. 1989; Nealson and Myers 1992). Thus, variations in the concentration of acetate and the variable $\mathrm{DO}_{2}$ uptake rates in shake flask cultures will cause a temporal shift in carbon source usage and electron flux. Such shifts can be expected to result in variable metabolic, transcriptomic and proteomic profiles between independent shake flask cultures if only a small number (1-3) of samples are taken, since one culture may have exhausted a carbon source and consequently shifted metabolism and electron flow while the "biological replicate" has not.
Given the general metabolic flexibility of bacteria including S. oneidensis MR-1 and their ability to respond rapidly to changes in environmental conditions, changes in the expressed proteins (proteome) would be expected as a result of exposing cultures to different environmental conditions. Through the use of comprehensive proteome measurements, the results presented here demonstrate that chemostat cultures provide the most consistent source of cellular material for biochemical measurements involving microbial cells with a minimum contribution to those measurements from biological variation. Two independent methods of proteome measurement, 2DE and AMT MS, both showed that flask cultures yielded protein samples with more culture-to-culture variability than observed in either controlled batch cultures or continuous chemostat cultures, primarily among proteins thought to be associated with anaerobic or microaerobic metabolism. A similar effect was found with respect to differential regulation of genes via microarrays when the results of two different investigations were compared from yeast cells, showing less discrepancy between chemostatic cultures (Daran-Lapujade et al. 2004).

Many of the proteins present in higher abundance in the aerobic shake flask cultures relative to the controlled batch or chemostat cultures in this study have previously been reported to be up-regulated in anaerobic compared to aerobic cultures (Beliaev et al. 2002, 2005; Fang et al. 2006). MtrA,B,C and OmcA are involved in metal-reduction in anaerobic cultures supplied with metals as terminal electron acceptors (Beliaev and Saffarini 1998; Beliaev et al. 2001; Myers and Myers 2002; Pitts et al. 2003) while the outer membrane protein $\mathrm{OmpW}$ and fumarate reductase have both been observed to be significantly more abundant in anaerobic cultures supplied with iron or fumarate as electron acceptor than in aerobic cultures (Fang et al. 2006). Thus, the shake flask cultures in the experiments described here must have included cells experiencing a lack of oxygen due to the nutrient depletion that is an inherent complication of such cultures, resulting in a greater range of expressed proteins.

Achieving a systems-level understanding of microorganisms, including the underlying metabolic and regulatory networks that control cell physiology and the ability to predict responses to perturbations, is indeed an ambitious goal. Systems biology takes full advantage of the state-of-thescience technology and genomic information, with the scientific as well as the practical benefits expected to be plentiful. Our results emphasize that functional genomics can greatly benefit from well-defined and homogeneous cell cultures provided by controlled cultivation techniques. The use of controlled cultivation techniques ensures that the large and comprehensive datasets used in systems biology research are derived from well-characterized and homogeneous 
biological samples, thus reducing the inherent variability that often complicates drawing biological conclusions and will allow for more accurate and robust identifications of metabolic and regulatory networks.

Acknowledgments This work was supported by the Genomics:GTL program of the U.S. Department of Energy's (DOE) Office of Biological and Environmental Research (OBER). We would like to thank the Microbial Cell Dynamics Laboratory (MCDL) at Pacific Northwest National Laboratory for use of the controlled cultivation technologies applied in this research. A portion of the research was performed at the W. R. Wiley Environmental Molecular Sciences Laboratory, a national scientific user facility sponsored by OBER and located at Pacific Northwest National Laboratory (PNNL). PNNL is operated for the DOE by Battelle Memorial Institute under Contract DE-AC0576RL01830. The 2DE work was performed at Argonne National Laboratory, operated by UChicago/Argonne LLC, under U.S. D.O.E Contract No. DE-AC02-06CH11357.

Open Access This article is distributed under the terms of the Creative Commons Attribution Noncommercial License which permits any noncommercial use, distribution, and reproduction in any medium, provided the original author(s) and source are credited.

\section{References}

Abboud R et al (2005) Low-temperature growth of Shewanella oneidensis MR-1. Appl Environ Microbiol 71:811-816

Adams CJ, Redmond MC, Valentine DL (2006) Pure-culture growth of fermentative bacteria, facilitated by $\mathrm{H}_{2}$ removal: bioenergetics and $\mathrm{H}_{2}$ production. Appl Environ Microbiol 72:1079-1085

Anderson NG, Anderson NL (1978a) Analytical techniques for cell fractions XXI. Two-dimensional analysis of serum and tissue proteins: multiple isoelectric focusing. Anal Biochem 85:331340

Anderson NL, Anderson NG (1978b) Analytical techniques for cell fractions XXII. Two-dimensional analysis of serum and tissue proteins: multiple gradient-slab gel electrophoresis. Anal Biochem 85:341-354

Beliaev AS, Saffarini DA (1998) Shewanella putrefaciens mtrB encodes an outer membrane protein required for $\mathrm{Fe}(\mathrm{III})$ and $\mathrm{Mn}$ (IV) reduction. J Bacteriol 180:6292-6297

Beliaev AS, Saffarini DA, McLaughlin JL, Hunnicutt D (2001) MtrC, an outer membrane decahaem c cytochrome required for metal reduction in Shewanella putrefaciens MR-1. Mol Microbiol 39:722-730

Beliaev AS et al (2002) Gene and protein expression profiles of Shewanella oneidensis during anaerobic growth with different electron acceptors. Omics 6:39-60

Beliaev AS et al (2005) Global transcriptome analysis of Shewanella oneidensis MR-1 exposed to different terminal electron acceptors. J Bacteriol 187:7138-7145

Blankenhorn D, Phillips J, Slonczewski JL (1999) Acid- and base-induced proteins during aerobic and anaerobic growth of Escherichia coli revealed by two-dimensional gel electrophoresis. J Bacteriol 181:2209-2216

Conrads TP, Anderson GA, Veenstra TD, Pasa-Tolic L, Smith RD (2002) Utility of accurate mass tags for proteome-wide protein identification. Anal Chem 72:3349-3354

Daran-Lapujade P, Jansen MLA, Daran J, vanGulik W, Winde JHd, Pronk JT (2004) Role of transcriptional regulation in controlling fluxes in central carbon metabolism of Saccharomyces cerevisiae. J Biol Chem 279:9125-9138
Dolla A, Pohorelic BKJ, Voordouw JK, Voordouw G (2000) Deletion of the hmc operon of Desulfovibrio vulgaris subsp. vulgaris Hildenborough hampers hydrogen metabolism and low-redox-potential niche establishment. Arch Microbiol 174:143-151

Elias DA, Suflita JM, McInerney MJ, Krumholz LR (2004) Periplasmic cytochrome $c_{3}$ of Desulfovibrio vulgaris is directly involved in $\mathrm{H}_{2}$-mediated metal but not sulfate reduction. Appl Environ Microbiol 70:413-420

Elias DA et al (2005) Global detection and characterization of hypothetical proteins in Shewanella oneidensis MR-1 using LC-MS based proteomics. Proteomics 5:3120-3130

Eng JK, McCormack AL, Yates JR III (1994) An approach to correlate tandem mass spectral data of peptides with amino acid sequences in a protein database. J Am Soc Mass Spectrom 5:976-989

Fang R et al (2006) Differential label-free quantitative proteomic analysis of Shewanella oneidensis cultured under aerobic and suboxic conditions by accurate mass and time tag approach. Molec Cell Proteomics 5:714-725

Gest H (1987) How microbes are isolated and identified, Chap. 5. In: Gest H (ed) The world of microbes. Science Tech Publishers, Madison, pp 33-38

Giometti CS, Taylor J (1991) The application of two-dimensional electrophoresis to mutation studies. In: Dunn MJ (ed) Advances in electrophoresis. Walter de Gruyter and Co, New York, pp 359389

Giometti CS, Gemmell MA, Tollaksen SL, Taylor J (1991) Quantitation of human leukocyte proteins after silver staining: a study with two-dimensional electrophoresis. Electrophoresis 12:536-543

Ji G, Beavis RC, Novick RP (1995) Cell density control of Staphylococcal virulence mediated by an octapeptide pheromone. Proc Natl Acad Sci USA 92:12055-12059

Keltjens JT, te BrSmmelstroet BW, Kengen SWM, vanderDrift C, Vogels GD (1990) 5,6,7,8-tetrahydromethanopterin-dependent enzymes involved in methanogenesis. FEMS Microbiol Rev $87: 327-332$

Kolkman A, Olsthoorn MMA, Heeremans CEM, Heck AJR, Slijper M (2005) Comparative proteome analysis of Saccharomyces cerevisiae grown in chemostat cultures limited for glucose or ethanol. Mol Cell Proteomics 4:1-11

Kolkman A et al (2006) Proteome analysis of yeast response to various nutrient limitations. Mol Syst Biol 16:1-16

Lipton MS et al (2002) Global analysis of the Deinococcus radiodurans proteome by using accurate mass tags. Proc Natl Acad Sci USA 99:11049-11054

Lovley DR, Phillips EJP, Lonergan DJ (1989) Hydrogen and formate oxidation coupled to dissimilatory reduction of iron or manganese by Alteromonas putrefaciens. Appl Environ Microbiol 55:700706

Maier TM, Myers JM, Myers CR (2003) Identification of the gene encoding the sole physiological fumarate reductase in Shewanella oneidensis MR-1. J Basic Microbiol 43:312-327

Mayville P et al (1999) Structure-activity analysis of synthetic autoinducing thiolactone peptides from Staphylococcus aureus responsible for virulence. Proc Natl Acad Sci USA 96:1218-1223

McLean JS et al (2007) Transcriptome study of oxygen-dependent autoaggregation in the dissimilatory metal reducer Shewanella oneidensis MR-1. J Bacteriol (in press)

Miller MB, Bassler BL (2001) Quorum sensing in bacteria. Annu Rev Microbiol 55:165-199

Monod J (1950) La Technique de culture continue. Theorie et applications. Ann Inst Pasteur 79:390-410

Myers CR, Myers JM (1992) Fumarate reductase is a soluble enzyme in anaerobically grown Shewanella putrefaciens MR-1. FEMS Microbiol Lett 98:13-20

Myers CR, Myers JM (2002) MtrB is required for proper incorporation of the cytochromes OmcA and $\mathrm{OmcB}$ into the outer membrane of 
Shewanella putrefaciens MR-1. Appl Environ Microbiol 68:5585-5594

Narang A, Konopka A, Ramkrishna D (1997) New patterns of mixedsubstrate utilization during batch growth of Escherichia coli K12. Biotechnol Bioeng 55:747-757

Nealson KH, Myers CR (1992) Microbial reduction of manganese and iron: new approaches to carbon cycling. Appl Environ Microbiol 58:439-443

Nethe-Jaenchen R, Thauer RK (1984) Growth yields and saturation constant of Desulfovibrio vulgaris in chemostat culture. Arch Microbiol 137:236-240

Norbeck AD, Monroe ME, Anderson KK, Daly DS, Adkins JN, Smith RD (2005) The utility of accurate mass and LC elution time information in the analysis of complex proteomes. J Am Soc Mass Spectrom 16:1239-1249

Novick A, Szilard L (1950) Description of the chemostat. Science 112:715-716

O'Farrell PH (1975) High-resolution two-dimensional electrophoresis of proteins. J Biol Chem 250:4007-4021

Pin C, Garcia de Fernando GD, Ordonez JA (2002) Effect of modified atmosphere composition on the metabolism of glucose by Brochothrix thermosphacta. Appl Environ Microbiol 68:4441-4447

Piper MDW et al (2002) Reproducibility of oligonucleotide microarray transcriptome analyses. J Biol Chem 277:37001-37008

Pitts KE, Dobbin PS, Reyes-Ramirez F, Thomson AJ, Richardson DJ, Seward HE (2003) Characterization of the Shewanella oneidensis MR-1 decaheme cytochrome MtrA: expression in Escherichia coli confers the ability to reduce soluble Fe(III) chelates. J Biol Chem 278:27758-27765

Ramagli LS, Rodriguez LV (1985) Quantitation of microgram amounts of protein in two-dimensional polyacrylamide gel electrophoresis sample buffer. Electrophoresis 6:559-563

Sadygov RG et al (2002) Code development to improve the efficiency of automated MS/MS spectra interpretation. J Proteome Res $1: 211-215$

Sedgwick WT (1916) The genesis of a new science-bacteriology. J Bacteriol 1:1-4

Senko JM, Istok JD, Suflita JM, Krumholz LR (2002) In-situ evidence for uranium immobilization and remobilization. Environ Sci Technol 36:1491-1496

Shen Y et al (2001) High-throughput proteomics using high-efficiency multiple-capillary liquid chromatography with on-line high-performance ESI FTICR mass spectrometry. Anal Chem 73:3011-3021
Shen Y, Zhao R, Berger SJ, Anderson GA, Rodriguez N, Smith RD (2002) High-efficiency nanoscale liquid chromatography coupled on-line with mass spectrometry using nanoelectrospray ionization for proteomics. Anal Chem 74:4235-4249

Smith RD et al (2002) An accurate mass tag strategy for quantitative and high-throughput proteome measurements. Proteomics 2:513523

Snoep JL, Teixeira-deMattos MJ, Postma PW, Neijssel OM (1990) Involvement of pyruvate dehydrogenase in product formation in pyruvate-limited anaerobic chemostat cultures of Enterococcus faecalis. Arch Microbiol 154:50-55

Stancik LM, Stancik DM, Schmidt B, Barnhart DM, Yoncheva YN, Slonczewski JL (2002) pH-Dependent expression of periplasmic proteins and amino acid catabolism in Escherichia coli. J Bacteriol 184:4246-4258

Thompson DK et al (2002) Transcriptional and proteomic analysis of a ferric uptake regulator (fur) mutant of Shewanella oneidensis: possible involvement of fur in energy metabolism, transcriptional regulation, and oxidative stress. Appl Environ Microbiol 68:881892

Valentine N, Wunschel S, Wunschel D, Petersen C, Wahl K (2005) Effect of culture conditions on microorganism identification by matrix-assisted laser desorption ionization mass spectrometry. Appl Environ Microbiol 71:58-64

Vasconcelos I, Girbal L, Soucaille P (1994) Regulation of carbon and electron flow in Clostridium acetobutylicum grown in chemostat culture at neutral $\mathrm{pH}$ on mixtures of glucose and glycerol. J Bacteriol 176:1443-1450

Wan X et al (2004) Transcriptomic and proteomic characterization of the Fur modulon in the metal-reducing bacterium Shewanella oneidensis. J Bacteriol 186:8385-8400

Wimpenny JW (1985) Novel growth systems. Microbiol Sci 2:53-60

Wright JD, Holland KT (2003) The effect of cell density and specific growth rate on accessory gene regulator and toxic shock syndrome toxin-1 gene expression on Staphylococcus aureus. FEMS Microbiol Lett 218:377-383

Wunschel DS et al (2005) Effects of varied pH, growth rate and temperature using controlled fermentation and batch culture on matrix assisted laser desorption/ionization whole cell protein fingerprints. J Microbiol Methods 62:259-271

Yohannes E, Barnhart DM, Slonczewski JL (2004) pH-dependent catabolic protein expression during anaerobic growth of Escherichia coli K-12. J Bacteriol 186:192-199 